\title{
Turned in fasciocutaneous island flap from face and neck based on subdermal microcirculation for reconstruction of the oral cavity following cancer surgery
}

\author{
Kalpesh Jayantkumar Gajiwala \\ Holy Family Hospital, Bhatia Hospital, Saifee Hospital, Ramakrishna Mission Hospital, \\ Mumbai, Maharashtra, India
}

Address for correspondence: Dr. Kalpesh Jayantkumar Gajiwala, 801, Aqua, Planet Godrej, Keshavrao Khadye Road, Mahalaxmi, Mumbai - 400 011, Maharashtra, India. E-mail: gajiwalakalpesh@gmail.com

\section{ABSTRACT}

Background: The skin and soft tissues of the face and neck have a rich plexus of dermal-subdermal vessels, which creates the possibility of raising a fasciocutaneous flap based on this vascular supply. A turned in fasciocutaneous island flap (TIFCIF) from an adjacent area of the defect can provide a simple substitute to many complex reconstructive procedures. Materials and Methods: Fifteen patients underwent wide excision for oral cancer and upper neck dissection, maintaining bone framework, between August 2010 and June 2014. The reconstruction was done with a 'turned in fasciocutaneous island flap' (TIFCIF) from the upper neck-lower face based on subdermal superficial adipofascial microvascular network instead of a specific known feeder vessel. The upper cervical flap, the donor site, when raised during the initial incision and the upper neck dissection, was cut off from the facial artery, the submental artery, the superior thyroid and the transverse cervical arteries. The microcirculation depended largely on the rich vascular plexus formed of the branches of the superficial temporal and the maxillary arteries. The donor site was primarily closed in Fourteen of the Fifteen patients. Eight patients received post-surgery irradiation. Results: All the flaps survived and healed and withstood radiation well. Two patients had flap dehiscence from suture line, of which one needed reattachment while other a minor one healed by itself and one patient had occasional drooling. There was no difficulty in eating or swallowing. Conclusions: A random patterned TIFCIF based on superficial subdermal adipofascial microcirculation, formed of a rich vascular plexus in the face and neck region can provide a simple alternative for intraoral reconstruction.

\section{KEY WORDS}

A turned in fasciocutaneous island flap; oral cancer reconstruction; turned in fasciocutaneous island flap; vascularity of face

\begin{tabular}{|l|l|}
\hline \multicolumn{2}{|c|}{ Access this article online } \\
\hline Quick Response Code: & Website: \\
\hline & www.ijps.org \\
\cline { 2 - 2 } & Dol: \\
\hline
\end{tabular}

This is an open access article distributed under the terms of the Creative Commons Attribution-NonCommercial-ShareAlike 3.0 License, which allows others to remix, tweak, and build upon the work non-commercially, as long as the author is credited and the new creations are licensed under the identical terms.

For reprints contact: reprints@medknow.com

How to cite this article: Gajiwala KJ. Turned in fasciocutaneous island flap from face and neck based on subdermal microcirculation for reconstruction of the oral cavity following cancer surgery. Indian J Plast Surg 2016;49:362-9. 


\section{INTRODUCTION}

ral cancer accounts for $12.5 \%$ of overall cancers in India ${ }^{[1,2]}$ contributing significantly to the challenges faced by oncosurgeons and plastic surgeons. Based on the complexity, the reconstruction of buccal mucosa defect has been done with direct closure, ${ }^{[1]}$ skin grafting ${ }^{[3]}$ or various flaps, such as the pectoralis major myocutaneous flap (PMMC), once a workhorse for head and neck reconstruction, ${ }^{[4,5]}$ the free radial artery forearm flap (FRAFF), ${ }^{[2,5,6]}$ the forehead flap, ${ }^{[7]}$ the deltopectoral flap, ${ }^{[8,9]}$ the temporalis muscle flap and fascia, ${ }^{[10,11]}$ anterolateral thigh (ALT) flap ${ }^{[2]}$ and the platysma flap. ${ }^{[12-16]}$ However, many of these flaps may be bulky and require extensive surgery and time. Here, a simple new flap from the face and neck region is described based on the rich vascular plexus. It achieves its purpose with minimum time and effort and negligible side effects for the patient.

\section{MATERIALS AND METHODS}

A retrospective study of Fifteen patients between the age group of 26 and 68 years, operated for squamous cell carcinoma of oral cavity from August 2010 to June 2014 were included in the study. Patients with moderate defects of buccal mucosa, floor of mouth and labial mucosal defects were included in the study. All patients underwent modified radical neck dissection with preserved mandibular arch. Male:female ratio was $2: 1$.

\section{Surgical technique}

The flap is planned at the lower border of the lower face-upper cervical flap that is raised during neck dissection. The flap is usually vertically below the defect and involves creation of a random pattern island flap of skin and subcutaneous soft tissue based on a subdermal microcirculation of the superficial adipofascial layer. The pedicle is raised at the upper border of the island flap by subdermal dissection 1-2 mm below dermis or below the roots of the hair follicles up to about $1.5-2.5 \mathrm{~cm}$ with a wide base. Minimum pedicle width is about $50 \%$ of the length of the flap, preferably based in the middle of the flap. The flap is then 'turned in' and passed over the mandible, if needed through an adequately created tunnel, to reach the defect without any constriction of the pedicle to reconstruct the buccal mucosa defect.

Some representative cases are discussed here.

\section{Case 1}

A 65-year-old woman with a recurrent carcinoma of left buccal mucosa, who was earlier operated for tongue cancer with upper neck dissection, had a defect starting from the retromolar area to the lip along with rim excision of the mandible following excision of the left buccal mucosa lesion [Figure 1a and b]. The defect was measured $(7.5 \mathrm{~cm} \times 2.2 \mathrm{~cm})$ and then transferred to the lower border of the incision of the lower face-upper neck flap [Figure 1c]. The upper, anterior and posterior margins of the marked flap were incised till the depth of the dermis. The 'turned in fasciocutaneous island flap' was raised at the lower border of the upper cervical flap by subdermal dissection under magnification at the upper border of the island flap up to $1-1.5 \mathrm{~cm}$ away from the island. The anterior and posterior ends of the flap were divided. The flap was then turned in, over the mandibular rim into the defect [Figure 1d]. The posterior end was sutured to the retromolar region, the lower border of the flap was sutured to the upper cut margin of the defect and the anterior cut end was set in the anterior part of the defect, whereas upper border was sutured with inferior cut margin. It is worthwhile to note that all the direct arterial blood supply including the facial and submental arteries were cut off [Figure 1a]. The flap easily set into and healed well [Figure 1e], and the donor site was skin grafted as a precaution to prevent tension on the pedicle (tumour-node-metastasis [TNM] staging: $T_{2}, N_{0}, M_{0}$ ). No post-surgery radiation was given. Patient healed well and is recurrence free.

\section{Case 2}

A 30-year-old woman with a post-excisional unreconstructed defect was referred because of a rapid

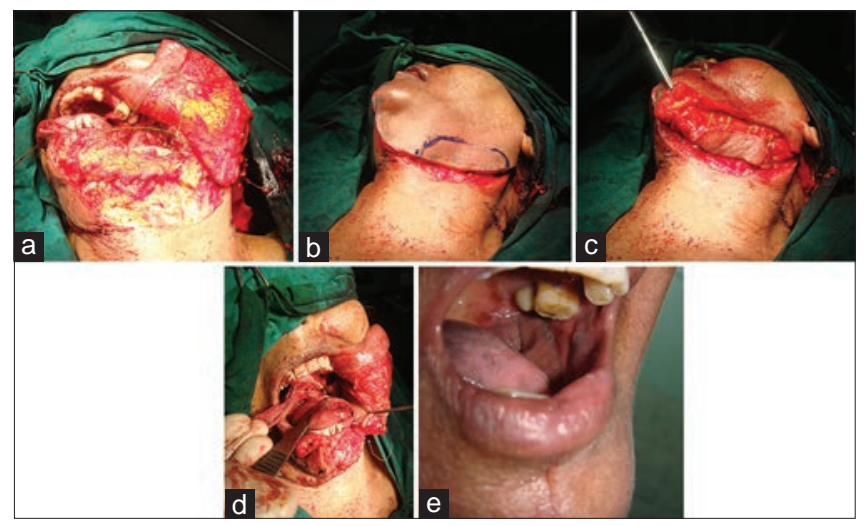

Figure 1: (a) The post-excision defect, as marked in green, of the left buccal mucosa and mandibular rim with lower face - upper neck skin flap completely lifted up off the mandible. There is no connection to the facial artery or its submental branch. (b) The island flap marked at the lower border of the cervical flap. (c) Upward subdermal dissection done at the upper border of the island flap. (d) The flap turned in and inset into the defect. Note the arc of rotation and the arrow pointing at the pedicle. (e) The flap post-healing 
recurrence [Figure 2a]. The oncosurgeon did a wide excision and radical neck dissection [Figures 2b]. The defect involved a part of the upper lip, buccal mucosa and lower lip. This time the island turnover flap to be raised was anterior starting from the midline. The lower facial skin was detached from the mandible, and the residual mental nerve branches and any arterial twig to the skin were divided [Figure $2 \mathrm{c}$ and $\mathrm{d}$ ]. The flap as marked was divided to the depth of the dermis and $5 \mathrm{~mm}$ subdermal dissection was done to create edges for the inner and outer lining [Figure 2e and f]. The flap was turned into cover the buccal defect as well as for the buccal lining of the lip [Figure $2 \mathrm{~g}$ and $\mathrm{h}$ ]. The neck side of the incision was closed primarily. The submental zone had a residual defect. This was closed by creating a small z-plasty. The flap showed post-operative congestion and there was a small wound gap [Figure 2i] at the lip but eventually healed well [Figure 2j] (TNM staging: $\mathrm{T}_{3}, \mathrm{~N}_{1}, \mathrm{M}_{0}$ ) Post-operative radiation was recommended; unfortunately, patient did not undergo radiation. Patient developed metastasis and died after 2 years.

\section{Case 3}

A 57-year-old male came with carcinoma of right buccal mucosa. He underwent radical neck dissection and wide excision. He had a large buccal mucosa defect starting from retromolar region upto lip involving partial loss of right upper lip, lower lip, commissure and part of cheek [Figure 3a and b]. A flap of $4.75 \mathrm{~cm} \times 11 \mathrm{~cm}$ length was raised at the lower border of the upper cervical flap from the angle of mandible to the chin [Figure 3c]. The island was demarcated and incised, only dermis deep, at the upper border. The subdermal dissection was then carried out up to about $1.5-2 \mathrm{~cm}$ upwards at the upper border [Figure 3d]. Both anterior and posterior subdermal pedicles were divided to keep about $50 \%$ of the central width of the pedicle. The flap was then turned in over the mandible [Figure 3e]. The posterior end was stitched to the retromolar defect. The lower border of the flap was stitched to the upper edge of the defect and upper border at the lower edge of the defect till the commissure [Figure 3f]. At the commissure, as seen two vertical cuts were made at upper and lower borders up to the dermis [Figure $3 \mathrm{~g}$ ]. A subdermal dissection was carried out up to $5 \mathrm{~mm}$ under both the edges to create cut edges for mucosa and the skin of the lips. A central undivided portion mimicked the commissure. Then the flap was turned outwards and back from the lip edge to fill the outer defect [Figure $3 \mathrm{~h}$ and i]. The flap was sutured. Patient received full course of radiation, and the flap survived and the patient is tumour free [Figure $3 \mathrm{j}$ and $\mathrm{k}$ ].

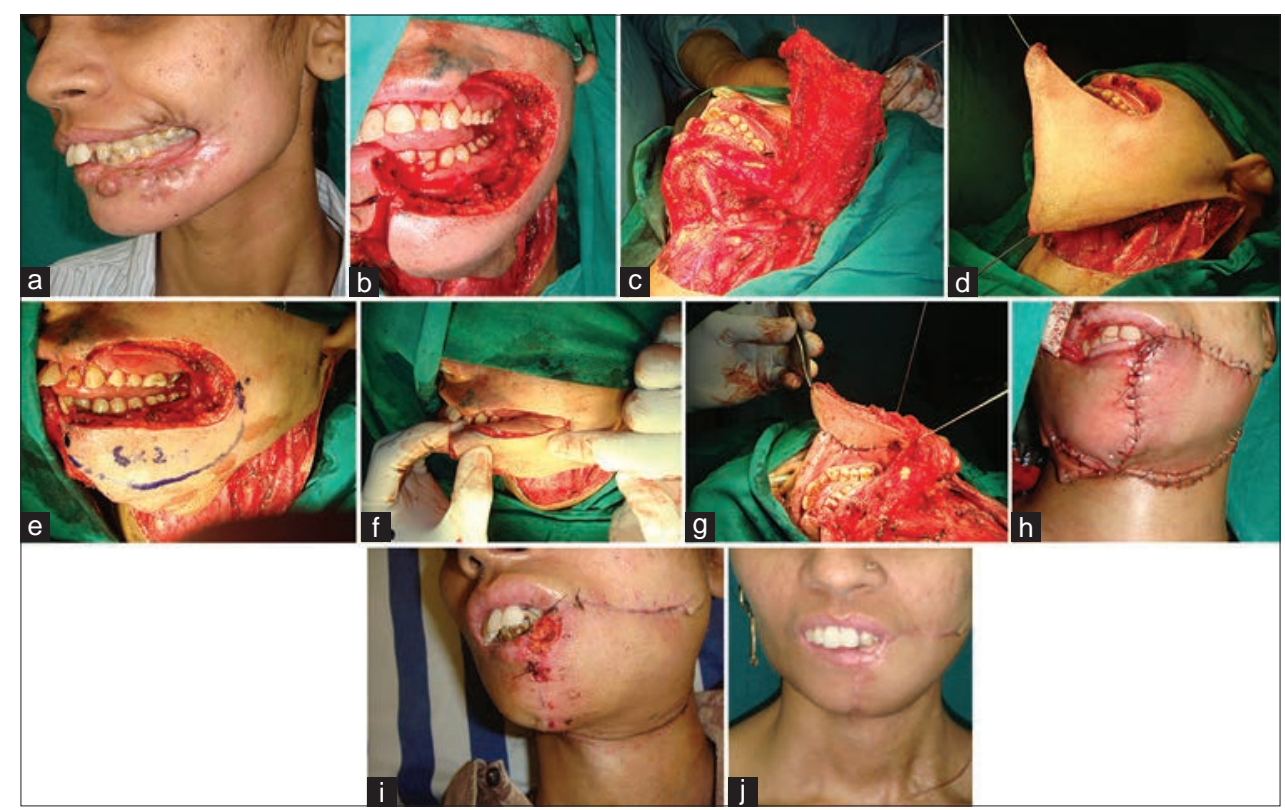

Figure 2: (a) A case of recurrent carcinoma of buccal mucosa and cheek with unreconstructed defect. (b) Same in front view, excision up to retromolar region. (c) The upper neck - lower face skin flap completely lifted up off the mandible, which removes any possible regular platysma blood supply. (d) The same upper neck - lower face skin flap, a donor site, external view. (e) The island flap created at the upper and anterior border of the above flap, just below the defect. (f) The island flap turned into create buccal lining. (g) The turned in part inset into the defect for the buccal lining. (h) Post-reconstruction external view, see tip congestion. (i) Wound dehiscence, not necrosis, at the lower lip. Maybe one can avoid anterior part of the incision in the flap which reconstructed the lower lip. (j) Following complete healing. Six months post-operative 


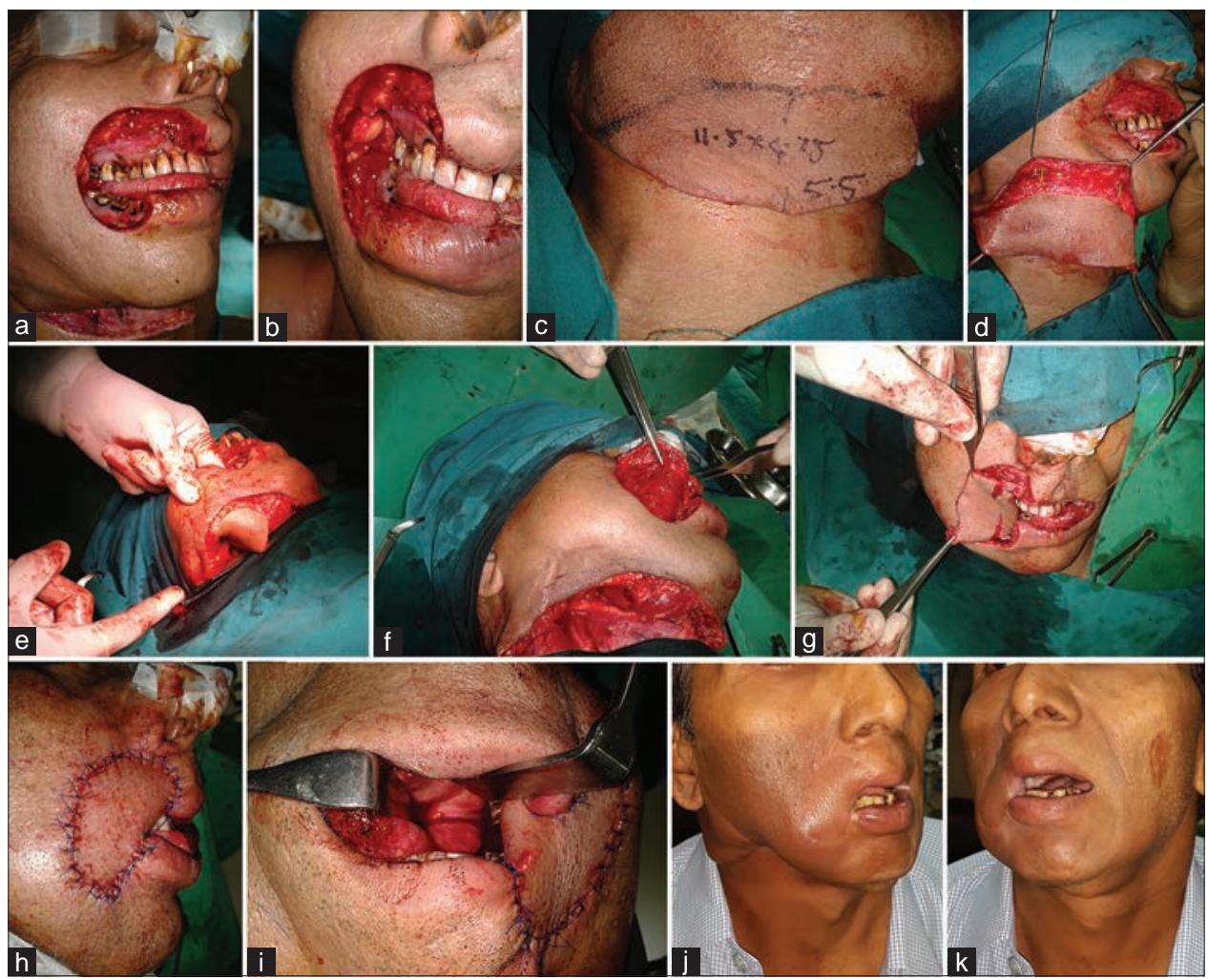

Figure 3: (a) A lateral oblique view of a defect following excision of carcinoma buccal mucosa, with removal of a part of the right upper lip, commissure, lower lip. (b) Front view, showing removal of entire buccal mucosa up to retromolar area. (c) $11 \mathrm{~cm} \times 4.75 \mathrm{~cm}$ island flap planned at the lower border of cervical flap. (d) Subdermal superficial adipofascial pedicle width is about $50 \%$ of the width of the flap, as marked by the green lines. (e) The island flap turned in through the tunnel. (f) The island flap sutured in the buccal mucosa defect and anterior portion brought out. (g) The anterior TIFCIF is turned outwards at commissure. Now, it becomes a turned in turned out fasciocutaneous island flap, vertically divided at the upper and lower border up to the depth of dermis, so as to insert it in the upper and lower lip edges. The central uncut portion forms the commissure. The microcirculation continuity remains as it is. (h) Immediate post-operative lateral view. (i) Immediate post-operative intraoral view, the commissure and insetting into the upper lip is quite visible. (j) Post-radiation therapy, a right-lateral oblique view. (k) Post-radiation intraoral view

\section{Case 4}

A55-year-old male came with carcinoma of left lower lip and buccal mucosa [Figure 4a and b]. He received neoadjuvant therapy and was operated with wide excision and upper neck dissection. He had a large defect involving lower lip, buccal mucosa, commissure and a small part of upper lip [Figure 4c]. Here, the island flap was raised anteriorly from below the defect [Figure 4d]. Anterior part was folded upon itself and turned in [Figure 4e] to create the lower lip including labial mucosa, commissure and to cover the cheek buccal mucosa defect [Figure $4 \mathrm{f}$ and $\mathrm{g}$ ]. Wound dehiscence occurred following early suture removal [Figure 4h]. A new incision was taken about $2 \mathrm{~cm}$ above the previous incision for reinsetting the flap at the upper border of the defect [Figure $4 \mathrm{i}$ and $\mathrm{j}$ ] and further healing was uneventful. Postoperatively, patient received full course of radiation [Figure $4 \mathrm{k}$ and $\mathrm{l}$.

\section{Case 5}

A case of recurrent right buccal carcinoma previously skin grafted, now involving lower lip, vermilion and part of right buccal mucosa, who had undergone right upper neck dissection earlier, was operated [Figure 5a and b]. Entire lower lip mucosa was removed with removal of most of the vermillion and part of right buccal mucosa by splitting the lower lip and chin down in the middle [Figure 5c]. Left side upper neck dissection was done. Here, an island flap from submental region based on subdermal microcirculation, and not on submental vessel, was raised with pedicle based laterally on the right side much above the mandibular margin [Figure $5 \mathrm{~d}-\mathrm{g}$ ]. The flap was then turned in to cover the lip vermillion and mucosal defect, and the skin below the vermilion on the right side was left in continuity with the cheek [Figure $5 \mathrm{~h}$ and i]. Patient received full course of radiation. The flap survived and there was no recurrence [Figure $5 \mathrm{j}$ and $\mathrm{k}$ ].

\section{RESULTS}

Fifteen patients were operated with this technique. Except for the first case, which was skin grafted, rest of the fourteen cases donor defects were closed primarily. 


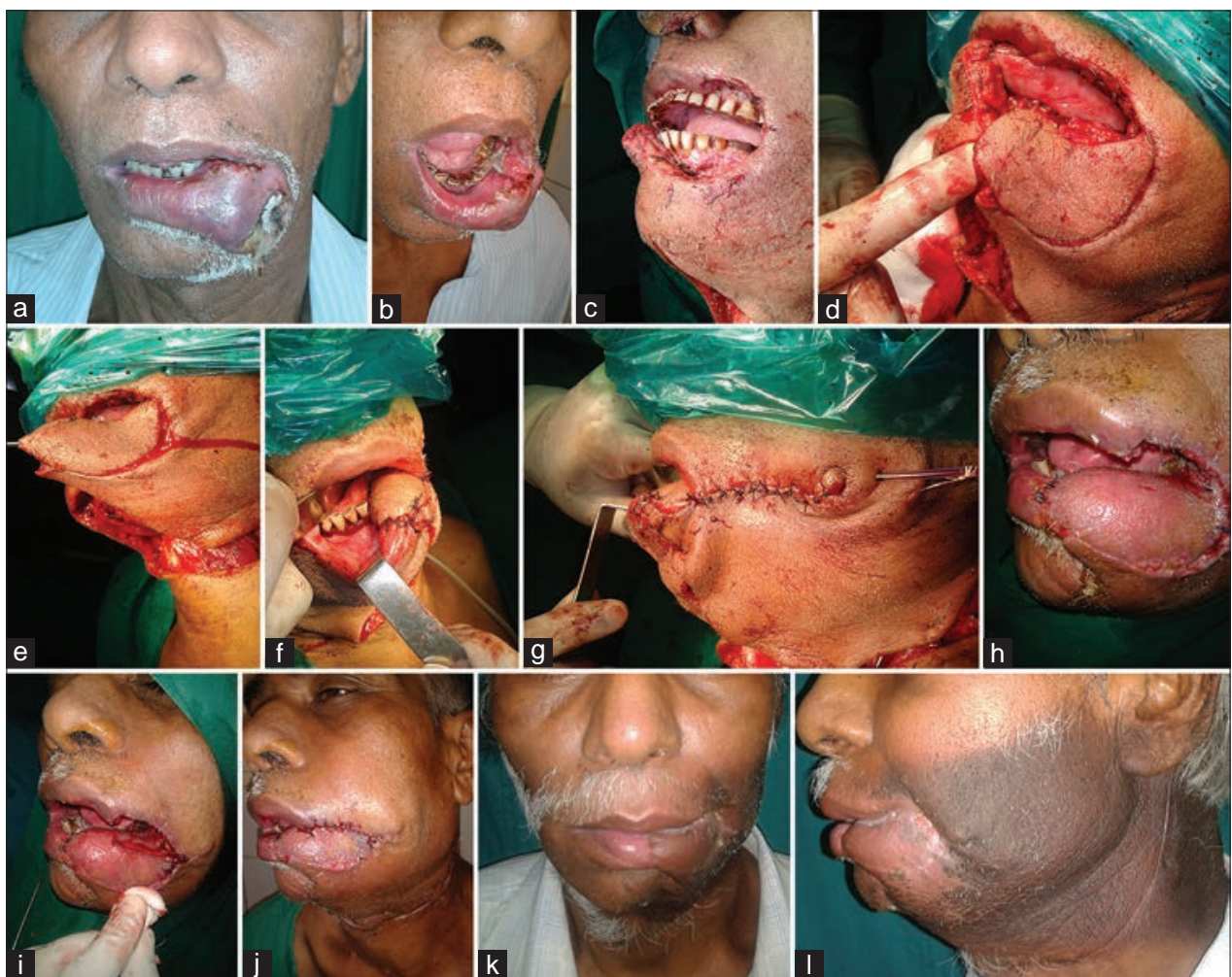

Figure 4: (a) Carcinoma of left lower lip and buccal mucosa, front view. (b) Intraoral view. (c) Post-excision defect. (d) TIFCIF planned vertically below the defect and incised up to the dermis. (e) Flap turned in to reconstruct buccal and labial mucosal defect. (f) Post-reconstruction intraoral view. (g) Post-reconstruction lateral view. (h) Wound gap at flap recipient junction following early stitch removal. Please note that the island flap incision line has receded downwards. (i) A fresh incision taken about two $\mathrm{cm}$ above the previous incision. (j) The new TIFCIF reattached (k) Post-irradiation, frontal view. (I) Post-irradiation lateral view. Even after second surgical stress the flap withstood irradiation

One case had a flap tip congestion and a slight dehiscence, which healed. One case had flap-recipient dehiscence due to inadvertent early suture removal. One patient had satellite recurrence, not involving flap and was treated with radiation. Two cases had minor donor defect wound healing problems. Two patients felt a little tightness in the neck, which resolved over few months. Eight patients were exposed to radiation and chemotherapy. There was no difficulty in swallowing or chewing with the remaining teeth.

\section{DISCUSSION}

During upper cervical lymph node dissection, a skin and soft tissue composite upper cervical flap is raised by an incision extending from the midline of the lower lip over the chin going posteriorly about 3-4 cm below the mandibular rim and reaching up to the mastoid behind the ear. A turned in fasciocutaneous island flap (TIFCIF) is generally raised from the lower border of this flap by upward subdermal dissection, which depends entirely on its blood supply from subcutaneous adipofascial microcirculation, as all the cervical blood supply including facial artery and its submental branch are divided during neck dissection. Earlier studies by Houseman et al. ${ }^{[17]}$ who mapped the angiosomes of the head and neck, showed the anastomosis between branches of the facial, superficial temporal, ophthalmic, posterior auricular, occipital arteries in the face, scalp and neck creating a rich anastomotic network and choke vessels. They considered the platysma as a vascular link between the external carotid and the subclavian artery through the submental and the superior thyroid arteries above and the transverse cervical and the inferior thyroid arteries below, so as to be able to raise superiorly or inferiorly based platysma flaps. They also showed a dense venous network in the lower face with the majority of veins being avalvular or with osteal valves allowing bidirectional flow. The pedicled platysma flaps were considered to be based on an arterial blood supply to the platysma. ${ }^{[13-15,17]}$ Imanishi et al. ${ }^{[18]}$ put a question mark on platysma circulation. Moreover, their brilliant detailed study of the circulation of the skin and soft tissue of the face and neck explains the reason for the success of TIFCIF. In the article, they described three layers - the deep adipofascial layer, the platysma layer and the skin and superficial adipofascial layer and 

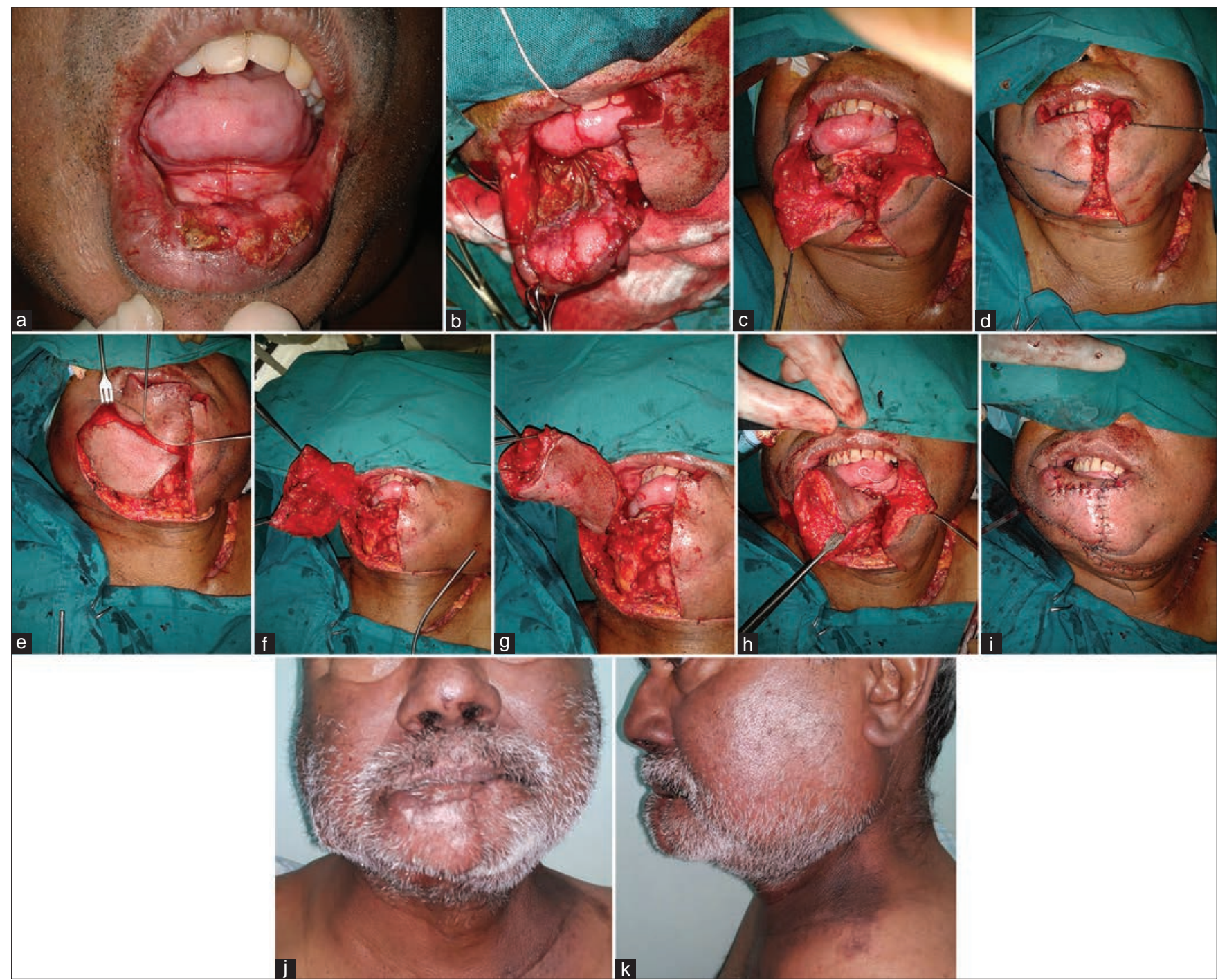

Figure 5: (a) A case of recurrent carcinoma lower lip, a satellite lesion, previously operated for right buccal mucosa with wide excision and upper neck dissection and reconstructed with skin grafting. (b) Involving the labial mucosa and vermilion. (c) Post-excision defect intraoral view. (d) Post-excision defect showing the extent of lower lip loss and marking the flap in the submental region. (e) The flap divided at the lower border and raised. (f) The flap is based on lateral subdermal superficial adipofascial pedicle. $(\mathrm{g})$ The lower half of the flap is tuned in, keeping the continuity of the skin and dermis from the cheek into the flap to avoid twisting of the pedicle. (h) The lower border of the flap is then incised dermis deep and then subdermally for about $5 \mathrm{~mm}$ on either side, so as to get lower margins to suture to gingival mucosa inside and skin outside. (i) The immediate post-operative view after the closure. (j) Post-radiation front view. (k) Post-radiation lateral view

observed that there was a rich subdermal-dermal plexus in the superficial adipofascial layer, and the plexus in the deep adipofascial layer was more significant with better vascular continuity than the platysma. They state, 'major vessels did not run long in the platysma layer. Any arterial branch of the anterolateral neck skin penetrated the platysma, and the platysma was supplied by only small vessels of the arterial branches. A vascular plexus was formed by those small vessels in the platysma, but the vascular density was low in comparison with that of the subdermal plexus. Furthermore, there were few vascular connections between the platysma plexus and the subdermal plexus. Accordingly, the platysma was only a plane where arteries for the skin penetrated, and it was difficult to say that the vascular system of the platysma became a source of blood supply to the skin'. ${ }^{[18]}$ Nonetheless, after conclusively establishing the importance of the robust microcirculation of superficial subdermal adipofascial plane, which was independent of platysma, they failed to extend its application to create a flap based on this microcirculation. TIFCIF should have been the logical end result. Instead they stopped short and concluded that the platysma flap should be called a fasciocutaneous flap, and that there is a possibility of raising a thin flap based on an arterial branch if one can find the artery which comes from the deep adipofascial plain, penetrates the platysma and runs along the superficial adipofascial plane. ${ }^{[18]}$ Thus, the TIFCIF as described above 
is based on a wide, but short pedicle of highly vascular superficial adipofascial tissue with a rich plexus of microcirculation. And the platysma is just an incidental part of the TIFCIF and more likely being sustained by the adipofascial vascular plexus rather than sustaining the overlying skin. ${ }^{[18]}$ The plexus supplying TIFCIF is formed from the anastomosis between the superficial temporal artery and branches from the maxillary artery mainly infraorbital and mental branch of inferior alveolar and a bit of cross supply from opposite facial through inferior and superior labial arteries if they are intact. The choke vessels and rich venous network allow the arterial territory of one angiosome to be freely supplied and drained by the vessels of another angiosome.

Clinically, therefore, TIFCIF appears to have robust microcirculation that withstands surgery, irradiation and chemotherapy well. After a subdermal dissection of $1-2 \mathrm{~cm}$ at the upper border of the flap, upward swing almost doubles the vertical length of the pedicle. The flap covers the area from retromolar region up to the midline. The flap can be divided and raised as more than one island flaps so long as the subdermal microcirculation is not disturbed. The flap can be used for both inner and outer lining. In case if the outer cheek defect occurs with a commissure being spared, the turned outwards and backwards part of the anterior portion of the flap can be deepithelialised in the intervening portion to inset the remaining flap in the outer defect. Thus, the TIFCIF provides effective cover for buccal mucosa, part of upper lip, lower lip, commissure, cheek, floor of the mouth, palate (in edentulous patient) and gingivobuccal sulcus. A moderate size defect as shown in Case 3 can be easily covered. As shown a minor dehiscence in one case and an unintended dehiscence in other case where a second incision within the previous flap was taken to successfully reattach the flap, points to the sturdiness of the circulation of TIFCIF. Although the subdermal adipofascial microcirculation is robust, the blood supply depends on the surface area and thickness of the bridge tissue. Moreover, an adequate tunnel to prevent pedicle compression and lack of tension would ensure high success rates. Since microcirculation is low-pressure slow flow system, tension particularly may be detrimental. When there is tension on a pedicle or a flap, the fine blood vessels in the microcirculation get stretched and squeezed by surrounding soft tissue, which causes the lumen to narrow and many a times changes the angles between two communicating vessels. The flow, which has an easy movement otherwise, now has to pass through narrowed channels at many acute angles leading to much slower flow and hypoxia or anoxia and flap necrosis.

Overall, the advantage of TIFCIF is that it is simple, safe, versatile, causes minimum blood loss and morbidity, requires much less surgical time and is sufficient for the modest defects of the most of the buccal mucosa, lower lip, floor of the mouth defect, oral commissure and part of the upper lip defect and cheek defect reconstruction and gives cosmetically acceptable results. It avoids secondary and tertiary defects, and morbidities associated with distant flaps such as FRAFF, PMMC flap, ALT flap, forehead flap and skin grafts. The limitations of TIFCIF are that it is only for the soft tissue reconstruction and cannot replace bone framework. It has a limited reach and size. The hair-bearing skin in males is a problem, which is also the case with FRAFF and PMMC. Moreover, in case where malignancy is suspected and cervical flap seems to be compromised one may not be able to use it. Furthermore, history of previous radiation may have compromised microcirculation as it reduces over a period of time after irradiation due to endothelial damage, making the flap survival unpredictable.

\section{CONCLUSIONS}

Facial and neck skin are highly vascular due to a rich subdermal and adipofascial vascular plexus. A simple random patterned turned in fasciocutaneous island flap (TIFCIF ) from the lower face-upper neck region based on this plexus can provide an easy solution for reconstruction in oral carcinoma. It adds one more possible flap technique in the existing armamentarium for reconstruction in the oral cavity.

\section{Financial support and sponsorship}

Nil.

\section{Conflicts of interest}

There are no conflicts of interest.

\section{REFERENCES}

1. Yadav P. Reconstructive surgery in oral cancers. Indian J Plast Surg 2007;40 Suppl S1:22-7.

2. Yadav P. Reconstructive surgery in oral cancers. Indian J Plast Surg 2013;46:275-82.

3. Sharp GS, Helsper JT. Intra-oral cancer. The use of skin grafts in the management of the condition. Calif Med 1964;100:332-5.

4. Aryan $\mathrm{S}$. The pectoralis major myocutaneous flap. A versatile flap for reconstruction in the head and neck. Plast Reconstr Surg 1979;63:73-81. 
5. Kuriakose MA, Sharma M, lyer S. Recent advances and controversies in head and neck reconstructive surgery. Indian J Plast Surg 2007;40 Suppl S1:3-12.

6. Bhathena HM, Kavarana NM. Bipaddled, extended radial forearm flap with microarterial anastomosis for reconstruction in oral cancer. JPRAS 1988;41:354-7.

7. Lewis MB, Remensnyder JP. Forehead flap for reconstruction after ablative surgery for oral and oropharyngeal malignancy. Plast Reconstr Surg 1978;62:59-65.

8. Bakamjian VY. A two-stage method for pharyngoesophageal reconstruction with a primary pectoral skin flap. Plast Reconstr Surg 1965;36:173-84.

9. Nayak BB, Nilamani M. Single stage reconstructions in head and neck surgery using deltopectoral and pectoralis major myocutaneous flaps. Indian J Plast Surg 2012;45:151-3.

10. Bradley $P$, Brockbank J. The temporalis muscle flap in oral reconstruction. A cadaveric, animal and clinical study. J Maxillofac Surg 1981;9:139-45.

11. Mokal NJ, Ghalme AN, Kothari DS, Desai M. The use of the temporoparietal fascia flap in various clinical scenarios: A review of 71 cases. Indian J Plast Surg 2013;46:493-501.

12. Futrell JW, Johns ME, Edgerton MT, Cantrell RW, Fitz-Hugh GS. Platysma myocutaneous flap for intraoral reconstruction. Am J Surg 1978;136:504-7.

13. Futrell JW, Robson JA. The platysma musculocutaneous flap: Experience with 24 cases. Plast Reconstr Surg 1983;72:322-3.

14. Hurwitz DJ, Rabson JA, Futrell JW. The anatomic basis for the platysma skin flap. Plast Reconstr Surg 1983;72:302-14.

15. Coleman JJ $3^{\text {rd }}$, Jurkiewicz MJ, Nahai F, Mathes SJ. The platysma musculocutaneous flap: Experience with 24 cases. Plast Reconstr Surg 1983;72:315-23.

16. Tessier P, Matthews DC, Kamerer D Jr., Ciminello FS, Gargano F, Wolfe SA. Platysma-based myocutaneous clavicular island flap for intraoral reconstruction. Ann Plast Surg 2011;67:S55-69.

17. Houseman ND, Taylor GI, Pan WR. The angiosomes of the head and neck: Anatomic study and clinical applications. Plast Reconstr Surg 2000;105:2287-313.

18. Imanishi N, Nakajima H, Kishi K, Chang H, Aiso S. Is the platysma flap musculocutaneous? Angiographic study of the platysma. Plast Reconstr Surg 2005;115:1018-24. 